\title{
Visual motion and attentional capture
}

\author{
ANNE P. HILLSTROM and STEVEN YANTIS \\ Johns Hopkins University, Baltimore, Maryland
}

\begin{abstract}
Previous work has shown that abrupt visual onsets capture attention. This occurs even with stimuli that are equiluminant with the background, which suggests that the appearance of a new perceptual object, not merely a change in luminance, captures attention. Three experiments are reported in which this work was extended by investigating the possible role of visual motion in attentional capture. Experiment 1 revealed that motion can efficiently guide attention when it is perfectly informative about the location of a visual search target, but that it does not draw attention when it does not predict the target's position. This result was obtained with several forms of motion, including oscillation, looming, and nearby moving contours. To account for these and other results, we tested a new-object account of attentional capture in Experiment 2 by using a global/local paradigm. When motion segregated a local letter from its perceptual group, the local letter captured attention as indexed by an effect on latency of response to the task-relevant global configuration. Experiment 3 ruled out the possibility that the motion in Experiment 2 captured attention merely by increasing the salience of the moving object. We argue instead that when motion segregates a perceptual element from a perceptual group, a new perceptual object is created, and this event captures attention. Together, the results suggest that motion as such does not capture attention but that the appearance of a new perceptual object does.
\end{abstract}

Motion, like many other visual features, is registered effortlessly by the human visual system. For example, discontinuities in motion can be detected in visual search without attentional scrutiny (McLeod, Driver, \& Crisp, 1988; Nakayama \& Silverman, 1986), just as salient discontinuities in color and orientation can be (see, e.g., Treisman \& Gormican, 1988). These findings suggest that visual motion can be detected without attention; motion is therefore often categorized as one of the "building blocks" of early vision. This has contributed to the belief that attention is involuntarily captured by moving objects: A natural way to draw someone's attention is to wave one's arms, and William James listed "moving things" among the sensorial stimuli to which attention is drawn involuntarily (James, 1890/1950, p. 417). Yet although findings support the claim that motion can be detected effortlessly when it is the target of search, there currently exists no direct evidence about whether motion captures attention.

The argument that motion captures attention runs as follows: (1) Attributes that can be detected without attentional scrutiny (i.e., attributes that "pop out" of a dis-

This research was supported by National Institute of Mental Health Grant R01-MH43924 to Steven Yantis. Portions of this research were reported at the 1992 meeting of the Eastern Psychological Association in Boston and at the 1992 meeting of the Psychonomic Society. Bill Bacon, Howard Egeth, and Brad Gibson provided valuable suggestions concerning the research. We thank Lara Dhingra, Jacqueline Farris, Robert Gaudet, Dawn Gondoli, Paul Hanna, Daphne Hu, Rachel Pointsett, Nitin Seam, John Tsouris, Rich Tyson, Lisa Yacono, and Marney Zell for excellent technical assistance. Correspondence may be sent to either author at the Department of Psychology, Johns Hopkins University, Baltimore, MD 21218. play) capture attention; (2) motion can be detected without attentional scrutiny; (3) therefore, motion captures attention. However, Premise 1 of this argument does not hold in general. To see why, one must draw a distinction between voluntary, goal-directed attentional guidance and stimulus-driven attentional capture (see, e.g., Yantis, 1993a). Goal-directed attentional guidance is revealed by experiments in which subjects allocate attention on the basis of task instructions and knowledge about current environmental contingencies. For instance, an informative spatial cue presented in advance of a visual display makes search more efficient (see, e.g., Posner, Snyder, \& Davidson, 1980), and knowledge that a salient feature distinguishes the target from all distractors in a display yields response times (RTs) that do not increase with the number of display elements (see, e.g., Treisman \& Gelade, 1980). In these cases, subjects' knowledge of the relationship between the location of the target and various other attributes of the display can be used to direct attention efficiently.

Stimulus-driven attentional capture, in contrast, is said to occur when attention is drawn to an attribute that is independent of or irrelevant to the subject's task. For instance, an invalid peripheral cue slows search for a target even when subjects know that the position of the cue is uncorrelated with the position of the target (Jonides, 1981). Yantis and Jonides (1984) found that an object that appears abruptly in a previously blank location draws attention even when the abrupt onset element is no more likely to be a visual-search target than any other element in the display.

Almost all reported examples of efficient detection of an object or attribute in visual search are instances of goal- 
directed attention allocation. For example, a feature singleton (e.g., a unique red element among green elements) is detected rapidly in search, but only if observers adopt a deliberate attentional set for singletons, or enter what Bacon and Egeth (in press) call "singleton detection mode." When a search display contains a feature singleton that is no more likely to be the target than any other element in the display, it does not capture attention. For example, Jonides and Yantis (1988) compared the visual search performance of three groups of subjects looking for a prespecified target element among varying numbers of nontarget elements. In one group, there was a uniquely colored element in each display (e.g., a red letter among green ones); in a second group, there was a single bright element among dim ones on each trial; in a third group, one element appeared abruptly in a previously blank location (an onset) among multiple no-onset elements (created by removing camouflaging line segments from block figure eight placeholders). In all three cases, the position of the unique element was uncorrelated with the position of the target. In these circumstances, only the onset element captured attention; the highly salient color and brightness singletons did not. Corroborating results have been reported by Theeuwes (1990).

These results show that features that can be detected without attentional scrutiny do not necessarily capture attention in a bottom-up or stimulus-driven fashion. Therefore, for one to determine whether motion captures attention in a purely bottom-up fashion, it is not enough to know that motion can be detected without attentional scrutiny. One must show that motion draws attention even when it is irrelevant to the observer's current perceptual task.

The hypothesis that motion captures attention is motivated in part by the common knowledge that motion is an effective way of drawing someone's attention, and in part by the similarity between motion and onsets (both involve the appearance of stimulus energy in a previously blank location). These considerations lead us to expect that motion will capture attention even when it is not part of the observer's attentional set. We tested this hypothesis in Experiment 1 by examining the effect of a single moving element among stationary elements in visual search.

\section{EXPERIMENT 1}

In Experiment 1, exactly one element in each search array exhibited motion. In the motion-relevant condition, the target, if present, was always the element exhibiting motion. In the motion-irrelevant condition, the position of the target was uncorrelated with the position of the moving element. On the basis of previous reports (e.g., Nakayama \& Silverman, 1986), we expected subjects in the motion-relevant condition to use the motion to direct attention efficiently, yielding RTs that would be independent of display size. This condition thus provided a way to ensure that the motion we employed was salient enough to guide attention when it was relevant.
The motion-irrelevant condition provided a strong test of the hypothesis that motion captures attention. In this condition, the moving element was the target on $1 / d$ of the trials in each condition, where $d$ is display size. If motion captures attention, we should observe display-size functions with zero slopes in the motion-irrelevant condition, where there is no incentive to deliberately attend to motion.

Five implementations of motion were tested in this way. These included three types of motion in which the contours of the letter remained stationary, but elements or textures within or near the letter moved: (1) Diagonally striped texture inside the moving element moved smoothly from right to left; (2) dots revolved around the moving element; or (3) random dots inside the moving element rapidly and randomly repositioned (producing scintillation). Two types of whole-letter motion were also used: (4) horizontal oscillation; and (5) looming (in which the moving element alternately grew large and small).

\section{Method}

\section{Subjects}

Ninety-three Johns Hopkins University students (52 male and 41 female) participated in this experiment for $\$ 5$ or to fulfill a research participation requirement for an introductory psychology course. In the motion-relevant condition, 14 subjects participated in the streaming-texture motion condition, and 5 in each of the other four motion conditions; in the motion-irrelevant condition, 11 subjects participated in the looming motion condition, and 12 in each of the other four motion conditions. No subject had participated in a related experiment before participating in this one, and no subject participated in more than one of the between-subject conditions of this experiment.

\section{Equipment and Stimuli}

The stimuli were displayed on a Taxan UV1150 21-in. color monitor controlled by an Artist Graphics XJS-1280 graphics board in a 386-based computer. The subjects responded by pressing buttons mounted on a response panel sitting on the table in front of them. A chinrest was used to maintain a constant viewing distance of $61 \mathrm{~cm}$.

Each display contained 4,10 , or 16 letters randomly positioned in a $6 \times 6$ array of positions and shifted slightly (up to $0.8^{\circ}$ horizontally and vertically) from base positions to avoid global configuration effects. The letter and array dimensions varied between implementations of motion. Table 1 lists the stimulus dimensions for the various motion types. Figure 1 shows a schematic representation of the layout of a display-size 10, target-present display in the streaming-texture condition. In one randomly selected position of each display, there was apparent motion, described below. The stimulus elements were block Ls and Ts that were upright or rotated $90^{\circ}, 180^{\circ}$, or $270^{\circ}$. The background of the display was always black. Schematics of sample elements are shown in reverse contrast in Figure 2: Stationary stimulus elements are shown in the top row and a representation of the type of motion is shown in the bottom row. The figures are not drawn to scale.

Streaming texture. The letters were outlined in white with a texture of black and white diagonal stripes inside that were $0.2^{\circ}$ wide, measured horizontally.

The motion was created by displacing the diagonal stripes $0.02^{\circ}$ to the left every $16.7 \mathrm{msec}$ within the area defined by the outline of the block letter until a response was made. Thus, the stripes appeared at the right edges of the letter and moved smoothly at a rate of $1.3 \% \mathrm{sec}$ until reaching the left edges of the letter. The moving stripes had the appearance of a revolving barbershop pole. 
Table 1

Stimulus Dimensions (in Degrees of Visual Angle) in Experiment 1

\begin{tabular}{|c|c|c|c|c|c|}
\hline \multirow[b]{2}{*}{ Motion Type } & \multicolumn{2}{|c|}{ Array } & \multirow{2}{*}{$\begin{array}{c}\text { Letter } \\
\text { Height/Width }\end{array}$} & \multirow{2}{*}{$\begin{array}{l}\text { Stroke } \\
\text { Width }\end{array}$} & \multirow{2}{*}{$\begin{array}{c}\text { Minimum } \\
\text { Inter-Element } \\
\text { Distance }\end{array}$} \\
\hline & Height & Width & & & \\
\hline Streaming texture & 20.7 & 19.4 & 2.1 & 0.6 & 1.2 \\
\hline Revolving dots & 20.7 & 19.4 & 2.1 & 0.6 & 1.2 \\
\hline Scintillation & 14.3 & 15.4 & 2.1 & 0.6 & 0.1 \\
\hline Oscillation & 16.0 & 15.1 & 1.6 & 0.1 & 0.7 \\
\hline Looming & 20.7 & 19.4 & $2.1 / 1.6^{*}$ & $0.6 / 0.5^{*}$ & 1.2 \\
\hline
\end{tabular}

*The first measurement in each pair is for stationary elements and the larger size of the looming element. The second measurement is for the smaller size of the looming element.

Revolving dots. The letters were black and were outlined in white. Surrounding each letter were four small, white squares evenly spaced on the circumference of an invisible circle of radius $1.6^{\circ}$ centered on the letter, positioned beginning at about $80^{\circ}$ clockwise from vertical. Each square was $0.2^{\circ} \times 0.2^{\circ}$ in size.

The squares revolved clockwise around one of the letters at a rate of 0.71 revolutions $/ \mathrm{sec}$ and were displayed at 12 positions in one revolution. They were displayed and stationary for $83 \mathrm{msec}$ at a time and erased for the next $33 \mathrm{msec}$ before being displayed again at the next position 0.08 revolutions away, and so on until a response was made. The motion appeared smooth. The squares surrounding all of the remaining letters in the display were stationary and always positioned at $12,3,6$, and 9 o'clock around the letters.

Scintillation. The letters were outlined in white and filled with a random dot texture which was created by dividing each letter into $0.1^{\circ}$ squares that were randomly assigned to be black or white.

Scintillation was produced in the letter with motion by randomly reassigning the positions of the white squares in all remaining frames. All frames had the same number of white squares so that the mean luminance of the letter did not vary. There was actually a sequence of five frames that was displayed repeatedly; the repe-

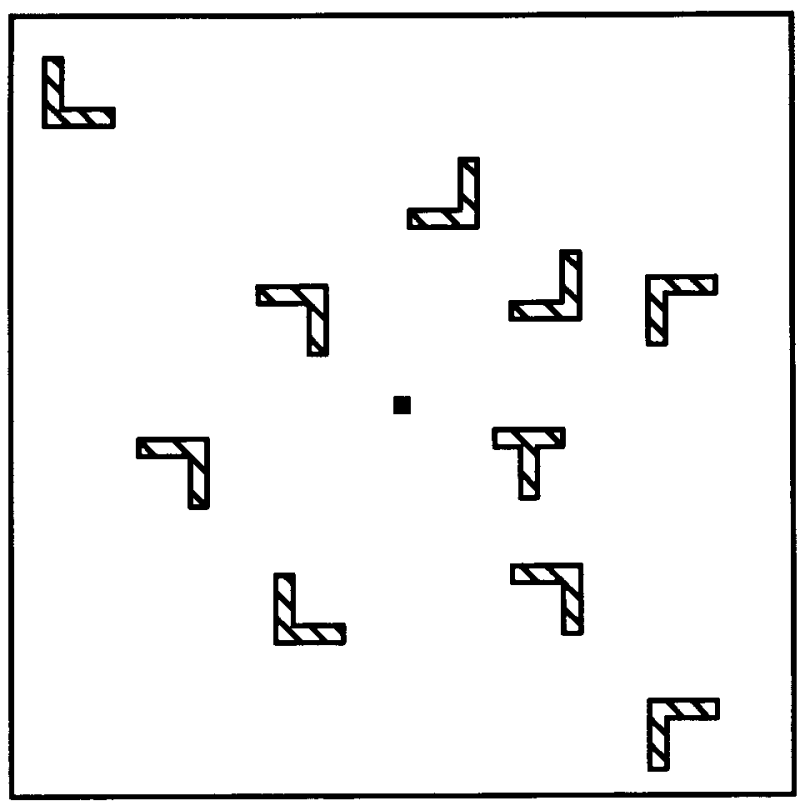

Figure 1. A sample display from the streaming texture condition of Experiment 1: Display Size 10, target present. tition was not evident to the viewer. Each frame was presented for $33 \mathrm{msec}$

Oscillation. The letters were solid white. Movement was created by displacing one letter $0.5^{\circ}$ horizontally in each frame, to the left in one frame, to the right in the next, and so on. The letter was present at the left location for $67 \mathrm{msec}$, was absent for the next $50 \mathrm{msec}$, was present at the right location for $67 \mathrm{msec}$, and was absent for $50 \mathrm{msec}$, and so on until a response was made.

Looming. The letters were black and were outlined in white. To create the looming at one location, two sizes of the same letter shape, centered at the same location, were alternated. The larger size was the same size as the stationary letters. The smaller size was $1.6^{\circ}$ in height with strokes $0.5^{\circ}$ wide. The letter was large for $83 \mathrm{msec}$, absent for $17 \mathrm{msec}$, small for $83 \mathrm{msec}$, absent for $17 \mathrm{msec}$, and so on until a response was made.

\section{Design}

There were two between-subject variables, motion type (streaming texture, revolving dots, scintillation, oscillation, and looming) and motion relevance (motion relevant or irrelevant to search), and two within-subject variables, display size $(4,10$, and 16$)$ and trial type (in the motion-relevant condition: target present and target absent; in the motion-irrelevant condition: target present/stationary, target present/moving, and target absent). The subjects completed 10 blocks of trials.

The total number of trials in each condition is shown in Table 2 . The target was present in half the trials and absent in the other half. In the motion-relevant condition, the target, when present, was always the moving element. In the motion-irrelevant condition, the moving element had a probability $1 / d$ of being the target, where $d$ is display size. The purpose of this aspect of the design, as stated earlier, was to ensure that there was no incentive for subjects to deliberately attend to the moving element. These proportions were achieved differently for different types of motion. In the revolvingdots condition, whether or not the target moved was determined randomly and independently for each trial, resulting in some random fluctuation in the proportion of target-moving trials between subjects. For the remaining types of motion, the number of trials in each condition was fixed at the values shown in Table 2 , so that given the total number of target-present trials for the display size, there were as close to $1 / d$ moving targets as possible.

\section{Procedure}

A small red fixation dot appeared in the middle of the screen throughout each block of trials. Each trial started when the fixation dot was displayed alone for $2 \mathrm{sec}$. Then the search array was displayed until the subject responded or until $5 \mathrm{sec}$ had elapsed without a response. The search array disappeared and the next trial began immediately after the previous trial ended. Motion began the moment the search array appeared and continued to the end of the trial. 


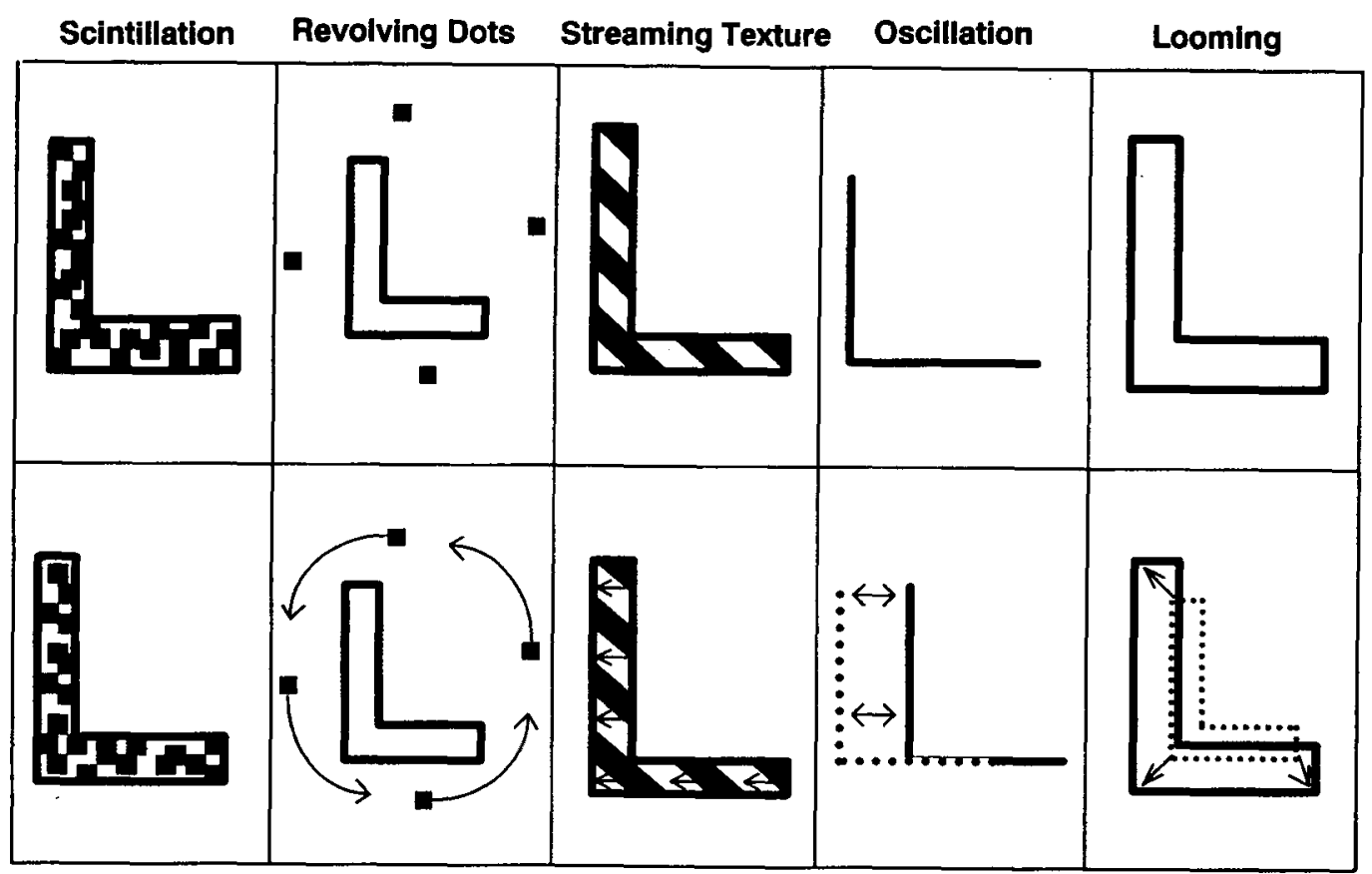

Figure 2. Stimuli from Experiment 1. (Schematics of the stimuli for the five types of motion. Top row: stationary elements. Bottom row: movement.)

On every trial, the subject searched for a $\mathrm{T}$, which could be rotated $0^{\circ}, 90^{\circ}, 180^{\circ}$, or $270^{\circ}$, among Ls, each at one of the four orientations, chosen randomly. If a $\mathrm{T}$ was present, the subject pressed the left response button with the left hand; if no $T$ was present, the subject pressed the right response button with the right hand.

The subjects in the motion-relevant group were told that the target, if present, was always the element with motion (of course, on half the trials the moving element was a nontarget). They were encouraged to use this to guide their search. The subjects in the motion- irrelevant group were informed that one element in each display would be moving, but that it was no more and no less likely to be the target than was any other element in the display. They were not specifically instructed whether or not to use the motion to guide their search. The subjects were told that when each trial began they should fixate on the central dot, but that eye movements were allowed once the search display appeared.

Each subject participated for one session that lasted about $45 \mathrm{~min}$. The session started with a practice block of about 15 trials. Each block began with 3 warmup trials. Each trial to which no response

Table 2

Number of Trials in Each Condition of Experiment 1

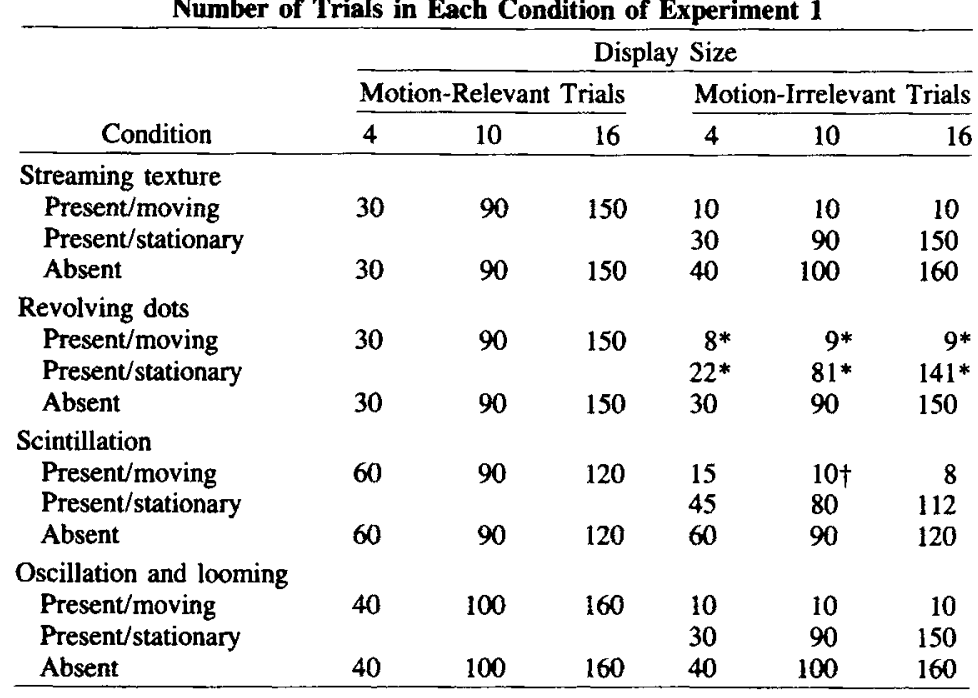

*These numbers are approximate. Each target-present trial was randomly assigned to a movement condition with probability $1 / d$ that the target would be moving, where $d$ is display size. TThis probability was inadvertently set to $1 / 9$ rather than $1 / 10$. 
or an incorrect response was made was followed by a feedback tone and a single recovery trial, chosen randomly from the trials constructed for the block. Data from the practice, warmup, and recovery trials were not analyzed. There was a short break after each block, lasting a minimum of $15 \mathrm{sec}$. The break after the fifth block lasted about $2 \mathrm{~min}$.

The subjects were told to respond as quickly as possible while maintaining accuracy: They were encouraged to make no more than two errors per block. If three errors were made within a single block, a message was displayed, encouraging the subject to slow down so as to improve accuracy. The message was repeated whenever another three errors were made in the same block. At the end of each block, a summary of the speed and accuracy for that block was displayed.

\section{Results and Discussion}

In this and all subsequent experiments reported in this article, outliers were removed from the data sets before the analyses were carried out. Outliers were defined as RTs faster than $300 \mathrm{msec}$ or slower than 3 standard deviations above the mean for that subject in that condition. This resulted in the removal of less than $2 \%$ of all observations. Very few trials were eliminated for being too fast.

All statistical tests reported in this paper were conducted with the use of a significance criterion of $p<.05$.

\section{Accuracy}

Table 3 shows the error rates in Experiment 1. The overall error rates were $3.7 \%, 3.5 \%, 3.2 \%, 2.2 \%$, and $2.4 \%$ for the streaming-texture, revolving-dots, scintillation, oscillation, and looming conditions, respectively. Analyses of variance (ANOVAs) of the error rates were carried out on motion-relevant and motion-irrelevant conditions separately. The ANOVA for the motion-relevant conditions included three factors: motion type, target presence, and display size. None of the main effects and none of the interactions were significant (all $F \mathrm{~s} \leq 1$ ). The ANOVA for motion-irrelevant trials, conducted only on data for target-present trials, had three factors: motion type, target motion, and display size. Error rates varied significantly with display size $[F(2,108)=8.9]$, but not with motion type or trial type $[F(4,54)=1.7$ and $F(1,54)=2.5]$. None of the interactions were significant [motion type $\times$ trial type, $F(4,54)<1$; motion type $\times$ display size, $F(8,108)<1$; trial type $\times$ display size, $F(2,108)=2.1$; and motion type $\times$ trial type $\times$ display size, $F(8,108)=1.2$ ].

Correlations between speed and accuracy were calculated for target-present trials to check for the possibility of speed-accuracy tradeoffs. Mean RTs and error rates were averaged across subjects for each combination of within-subject factors, resulting in six RTs and error rates to be correlated for each motion-relevant, motion-type condition, and nine pairs to be correlated for each motionirrelevant motion-type condition. Correlations for each of the 10 groups of subjects ( 5 motion types $\times 2$ motionrelevance conditions) ranged from $r(4)=0.95$ for relevant, revolving-dot motion to $r(4)=-0.74$ for relevant, streaming-texture motion. All but two of the correlations were negative, suggesting the possibility of a speed-accuracy tradeoff, but most of the correlations were small (between 0 and -.36 ) and none of the negative correlations were significant.

\section{Response Times}

Figure 3 shows the RTs for each condition of the experiment. Motion-relevant conditions are shown with dashed lines; motion-irrelevant conditions, with solid lines. Mean RTs for motion-relevant trials were analyzed with an ANOVA that included three factors: motion type, display size $(4,10$, or 16$)$, and target presence (present

Table 3

Error Rates (in Percent) for Each Condition of Experiment 1

\begin{tabular}{|c|c|c|c|c|c|c|}
\hline \multirow[b]{3}{*}{ Condition } & \multicolumn{6}{|c|}{ Display Size } \\
\hline & \multicolumn{3}{|c|}{ Motion-Relevant Trials } & \multicolumn{3}{|c|}{ Motion-Irrelevant Trials } \\
\hline & 4 & 10 & 16 & 4 & 10 & 16 \\
\hline \multicolumn{7}{|l|}{ Streaming texture } \\
\hline Present/moving & 3.1 & 2.0 & 3.1 & 5.0 & 4.4 & 5.8 \\
\hline Present/stationary & & & & 3.1 & 4.2 & 8.2 \\
\hline Absent & 1.9 & 1.8 & 2.8 & 0.9 & 2.2 & 0.9 \\
\hline \multicolumn{7}{|l|}{ Revolving dots } \\
\hline Present/moving & 0.7 & 1.3 & 2.5 & 3.0 & 1.6 & 7.5 \\
\hline Present/stationary & & & & 2.8 & 3.7 & 5.9 \\
\hline Absent & 1.3 & 2.9 & 3.2 & 0.3 & 0.2 & 0.1 \\
\hline \multicolumn{7}{|l|}{ Scintillation } \\
\hline Present/moving & 3.1 & 1.1 & 3.2 & 2.6 & 0.9 & 3.6 \\
\hline Present/stationary & & & & 2.9 & 4.5 & 7.3 \\
\hline Absent & 0.3 & 2.7 & 1.9 & 0.7 & 1.0 & 1.0 \\
\hline \multicolumn{7}{|l|}{ Oscillation } \\
\hline Present/moving & 1.5 & 1.6 & 1.3 & 4.2 & 1.7 & 0.0 \\
\hline Present/stationary & & & & 2.0 & 2.4 & 4.5 \\
\hline Absent & 2.1 & 1.2 & 1.0 & 0.6 & 0.6 & 0.9 \\
\hline \multicolumn{7}{|l|}{ Looming } \\
\hline Present/moving & 0.5 & 2.0 & 1.1 & 1.8 & 0.9 & 5.5 \\
\hline Present/stationary & & & & 2.5 & 3.4 & 3.5 \\
\hline Absent & 1.0 & 1.4 & 0.7 & 0.9 & 0.3 & 0.7 \\
\hline
\end{tabular}




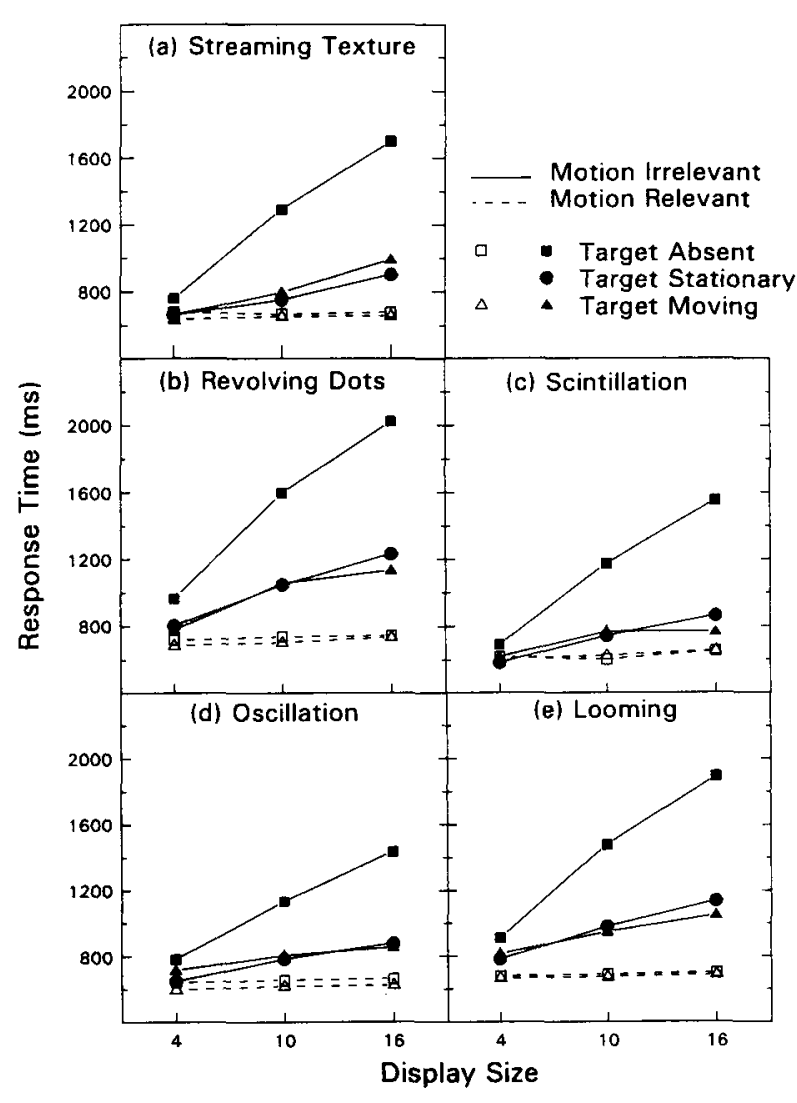

Figure 3. Results from Experiment 1. Response time is plotted as a function of display size for target-absent trials and for targetpresent trials with targets at the movement location and at stationary locations for the five types of motion: (a) Streaming texture. (b) Revolving dots. (c) Scintillation. (d) Oscillation. (e) Looming.

or absent). The main effect of motion type was not significant $[F(4,29)=1.4]$, nor were any of the interactions involving motion type $[F(8,58)=2.0$ for motion type $\times$ display size, $F(4,29)=1.0$ for motion type $\times$ target presence, and $F(8,58)<1$ for motion type $\times$ display size $X$ target presence]. Responses to target-present trials were significantly faster than responses to target-absent trials $[F(1,29)=7.68]$. Responses slowed significantly as display size increased $[F(2,58)=23.1]$. Display size did not interact significantly with target presence $[F(2,58)=1.5]$, as would be expected if the display-size effect was due to self-terminating, serial search through the display (Treisman \& Gelade, 1980). Rather, the pattern of effects is consistent with an effect like lateral inhibition, which should have increased equally for target-present and target-absent trials as target-distractor proximity increased with display size. The results are consistent with previous reports (e.g., Nakayama \& Silverman, 1986) that when motion is perfectly correlated with the position of the target, subjects can direct their attention to the moving element, make a presence/absence decision, and respond without scanning other elements in the display.

Mean RTs for target-present, motion-irrelevant trials were analyzed with an ANOVA that included three fac- tors: motion type, display size $(4,10$, or 16$)$, and trial type (moving or stationary). The main effect of motion type was significant $[F(4,54)=9.1]$; we will not discuss this further, because it is irrelevant to our arguments. The main effect of trial type was not significant $[F(1,54)<1]$, and trial type did not interact significantly with motion type $[F(4,54)=2.2]$. The main effect of display size was significant $[F(2,108)=180.0]$, and the interactions of display size with motion type and with trial type were significant $[F(8,108)=3.7$ and $F(2,108)=4.1$, respectively]. The three-way interaction of display size $\times$ motion type $\times$ trial type was not significant $[F(8,108)=1.2]$.

That the display-size effect was smaller for movingtarget trials than for stationary-target trials suggests there might have been some advantage due to target motion. However, two further findings eliminate the possibility that this advantage is strong attentional capture. If motion were capturing attention, we would expect no displaysize effect in the motion-irrelevant, target-moving condition. Table 4 lists the slopes of the display-size function for each condition of Experiment 1. Although the mean slope for motion targets in the motion-irrelevant condition is smaller than that for stationary targets (20.2 vs. $26.2 \mathrm{msec} /$ element), it is also significantly greater than zero $[t(58)=8.3]$. Furthermore, the moving-target slope in the motion irrelevant condition is also significantly greater than that in the motion-relevant condition (20.2 vs. $3.2 \mathrm{msec} / \mathrm{element})[t(61)=7.1]$. Instructions to use motion to guide search when motion provided useful information about target location significantly attenuated the display-size effect; this indicates that motion did not capture attention when it was irrelevant. Together, these results suggest that in motion-irrelevant conditions, subjects had to search the display for the target even when it was moving. The residual advantage for moving targets may reflect a strategic decision on the part of some subjects on some trials to voluntarily direct attention to the moving element. However, the hypothesis that the moving element captures attention, which would require slopes that are significantly attenuated and near zero, can be ruled out.

What cannot be ruled out at this time is the possibility that motion occasionally captured attention or that observers occasionally attended to the moving element during search earlier than would be expected by chance. If

Table 4

Slopes of the Display Size Functions (Milliseconds/Element) for Each Condition of Experiment 1

\begin{tabular}{|c|c|c|c|c|c|}
\hline \multirow[b]{2}{*}{ Motion Type } & \multicolumn{3}{|c|}{ Motion Irrelevant } & \multicolumn{2}{|c|}{ Motion Relevant } \\
\hline & $\begin{array}{l}\text { Target } \\
\text { Moving }\end{array}$ & $\begin{array}{c}\text { Target } \\
\text { Stationary }\end{array}$ & $\begin{array}{c}\text { Target } \\
\text { Absent }\end{array}$ & $\begin{array}{c}\text { Target } \\
\text { Moving }\end{array}$ & $\begin{array}{c}\text { Target } \\
\text { Absent }\end{array}$ \\
\hline reaming textu & $27.7 *$ & $22.7^{*}$ & $78.3 *$ & $4.06^{*}$ & 2. \\
\hline & & & & & 2.2 \\
\hline 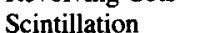 & 12 & 2 & 77 & $3.1^{*}$ & -0.3 \\
\hline sc & $11.6^{*}$ & 19. & 54 & $2.3^{*}$ & 1.8 \\
\hline Looming & $19.6^{*}$ & $29.6^{*}$ & $82.0^{*}$ & 2.3 & 2.6 \\
\hline$M$ & $20.2 *$ & $26.2 *$ & $76.0^{*}$ & $3.2 *$ & 1.8 \\
\hline
\end{tabular}

*Slope is significantly greater than zero. 
motion captured attention on some trials but not on others, attenuated but not flat slopes would be expected, and this is what we observed. This hypothesis could be evaluated in experiments designed to test for a mixture of trials in which attention was captured and trials in which it was not (Yantis, Meyer, \& Smith, 1991). The design of this experiment did not include enough trials to carry out such an analysis.

In summary, Experiment 1 yielded evidence that motion does not strongly capture attention. Contrary to the predictions of the attentional-capture model, display-size effects in target-moving, irrelevant-motion conditions were significantly greater than zero.

\section{EXPERIMENT 2}

How can we reconcile the results of Experiment 1 with the everyday knowledge that waving to a friend draws their attention? When someone is searching a crowd for a specific individual, he/she may adopt a deliberate attentional set for the attributes (such as motion) that are likely to distinguish the target (friend) from nontargets (strangers). The results of the motion-relevant condition of Experiment 1 demonstrated that such a strategy could be effective. Another possibility is that motion may be capturing attention only occasionally or partially.

A third possibility, which we examine in Experiments 2 and 3 , is motivated by our earlier finding that the onset of new perceptual objects, even in the absence of a luminance increment, captures attention (Yantis \& Hillstrom, 1994). In previous work on abrupt onsets, luminance increments and the appearance of new objects had always been confounded: All abrupt onsets were new objects accompanied by luminance increments. We unconfounded the two by conducting visual search experiments in which we used elements that were equiluminant with their backgrounds (elements defined by discontinuities in depth, motion, or texture) and found that new objects captured attention even in the absence of a luminance increment.

Although motion per se may not capture attention, as suggested by the results of Experiment 1, motion may play a role in causing the appearance of a new perceptual object, which in turn can capture attention. To make this idea concrete, consider the following examples.

The Geometridae moth, found in northern Borneo and elsewhere, has mottled wings that resemble the lichencovered bark on many trees in its niche. While the moth remains motionless, it is virtually invisible to predatory birds because of similarity grouping of the pattern on its wings with the pattern on the bark. As soon as its wings move, however, the moth instantly stands out as a new perceptual object. In this case, we would claim that it is not the motion of the wings per se that captures attention, but the appearance of the previously camouflaged perceptual object. Similarly, a motionless leopard blends in with the shadows and leaves of the jungle; as soon as it moves, the common fate of the leopard's spots define a new perceptual object that is easy to segregate from the background foliage. In these examples, occasional or partial capture seems an unlikely explanation, since it is not just attention that is affected by the motion, but the perceptual organization of the entire scene. In other words, even if attention is focused directly on the camouflaged object, it may not be seen until motion begins.

The new-object hypothesis is that motion (or any other attribute) captures attention when it requires the creation of a new object file. According to Kahneman and Treisman (1984; Kahneman, Treisman, \& Gibbs, 1992), an object file is a visual representation of a perceptual object containing specifications of the various attributes of the object, including its location, time of appearance, color, shape, and so forth. They have argued that the object file may form the basis of visual selection in many tasks. Other evidence for object-based theories of visual attention has been accumulating for the past several years (see Kanwisher \& Driver, 1992, for a recent review).

Our claim, then, is that when a new object file is created to accommodate the appearance of a new perceptual object, attention is directed there in a stimulus-driven fashion (see Van der Heijden, 1992, for a similar prediction). Our previous findings that abrupt visual onsets capture attention (e.g., Yantis \& Hillstrom, 1994; Yantis \& Johnson, 1990; Yantis \& Jonides, 1984) is consistent with this claim: An abrupt onset in a previously unoccupied location is clearly an instance of a new perceptual object.

In the motion-irrelevant conditions of Experiment 1, the elements were not densely packed and had no characteristics that would afford interelement grouping. A separate object file would therefore be established for each element at the moment it appeared, and the motion of one of the elements would be treated as an attribute of that object with no special attentional status. The moving element would therefore not be expected to capture attention. However, it is possible to construct visual tasks in which motion does set off an element from a perceptual group.

In Experiment 2, we used a global/local task (see, e.g., Navon, 1977, 1981) to simulate the case of the moth on the bark. The purpose of the experiment was to determine whether the appearance of a new object defined by motion would capture attention. The subjects were required to identify a global letter that was formed by a configuration of multiple small, densely packed, local letters (see Figure 4 for sample stimuli). On some trials, one of the local letters moved, which set that letter off as a separate perceptual object.

The global letter was always an $\mathrm{H}$ or an $\mathrm{S}$. All but one of the local letters were identical and response neutral (i.e., they were all the same letter other than $\mathrm{H}$ or S). The remaining unique local letter varied on two dimensions: response compatibility with the global letter, and temporal profile.

The identity of the unique local letter was compatible, neutral, or incompatible with the identity of the global letter (e.g., in the incompatible condition, if the global letter was an $\mathrm{H}$, the unique local letter was an S). Many previous experiments have shown that the response com- 


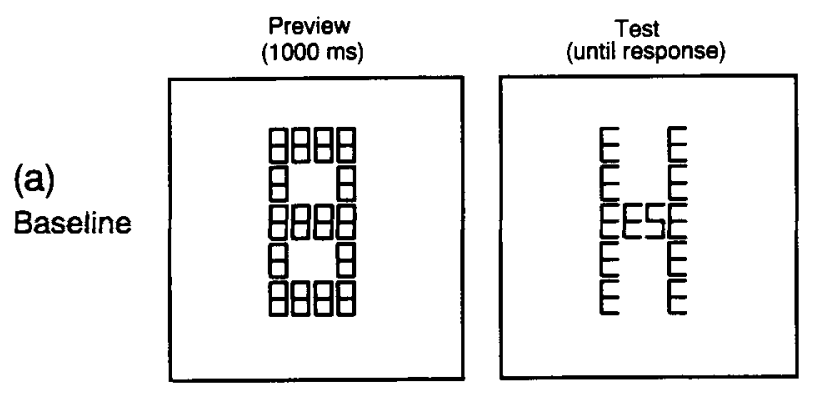

(b) Onset

(c) Late Motion Early Motion
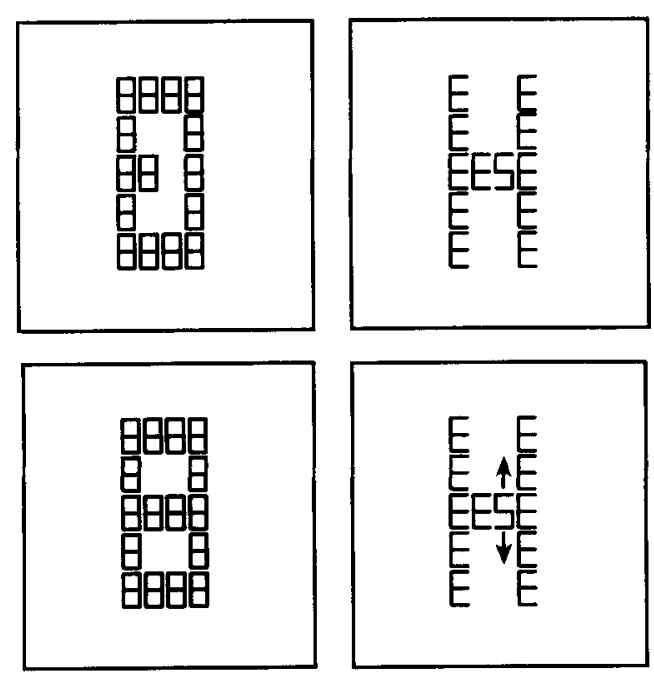
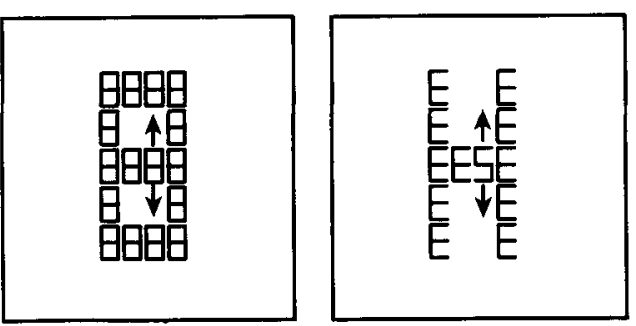

Figure 4. Displays used in Experiments 2 and 3. Panels a, b, and c show the baseline, onset, and motion conditions of Experiment 2, respectively; panel d shows the early-motion condition of Experiment 3. The left panel in each row shows the preview display, consisting of a global figure eight made up of local figure eights. The right panel in each row shows a sample target display, in which a global $H$ is made up of local Es, which are neutral, and a unique local S, which is incompatible with the global letter. The compatible and neutral conditions are not shown.

patibility of local letters can affect the speed of identifying global letters because of a failure of selective attention (Miller, 1981; Paquet, 1992; Paquet \& Merikle, 1988; Pomerantz, 1983; Pomerantz \& Sager, 1975; Shulman \& Wilson, 1987), although the more common result is that identification of the global letter remains uninfluenced by the local letters (e.g., Kimchi, 1992; Navon, 1977, 1981). In Experiment 2, our measure of attentional capture by the unique local letter was the magnitude of the observed response-compatibility effect. No effect of response-neutral local letters would be expected, whether or not attention were drawn to them. But if attention were drawn to a response-compatible or response-incompatible local letter, its effect should become stronger.

The second factor varied was the temporal profile of the unique local letter. The unique local letter was stationary (the baseline condition, as in Figure 4a), exhibited an abrupt visual onset (the onset condition, as in Figure 4b), or moved (the motion condition, as in Figure 4c). The new-object hypothesis predicts that attention should be drawn to the object segregated by motion, and so the magnitude of response-compatibility effects should be larger in the motion condition than in the baseline condition. The onset condition was included as a manipulation check: Our previous work strongly suggested that it should also produce substantial compatibility effects because it, too, involved the appearance of a new perceptual object.

\section{Method}

\section{Subjects}

Seventeen Johns Hopkins University students (11 females) participated in this experiment to fulfill research participation requirements for an introductory psychology class. No subject had previously participated in a related experiment. The data for 1 of the subjects were excluded from the analyses; the subject had RTs that were much slower and costs and benefits an order of magnitude larger than any other subject in the experiment. Excluding this subject's data did not qualitatively change the results.

\section{Equipment and Stimuli}

Fourteen of the 17 subjects viewed the displays on a Seiko Instruments model CM1450-C color monitor controlled by a Video Seven VRAM VGA graphics card. For 3 of the subjects, the stimuli were displayed on a CrystalScan model 1024 NI color monitor controlled by a SpeedStar Plus VGA graphics card. There were no measurable differences in the displays produced by these two systems. Both sets of subjects viewed the displays from a distance of about $60 \mathrm{~cm}$. No chinrest was used. The subjects responded by pressing buttons mounted on a response panel on the table in front of them.

Each trial consisted of three displays: the fixation display, the placeholder display, and the target display (see Figure 4). In the fixation display, a $0.6^{\circ}$ red crosshair appeared in the center of the screen for $500 \mathrm{msec}$, to orient the viewer to the center of the display and to provide warning that the trial was beginning. The placeholder display consisted of a global block figure eight made up of 16 local figure eights, 4 to a row and 5 to a column, that appeared for $1 \mathrm{sec}$. The global form was $2.2^{\circ}$ wide and $3.9^{\circ} \mathrm{high}$. The local forms were $0.4^{\circ}$ wide and $0.6^{\circ}$ high and were separated $0.6^{\circ}$ horizontally and $0.8^{\circ}$ vertically, measuring center to center. The local forms were drawn with solid strokes.

The target display, which was present until the subject responded, consisted of a global letter made up of local letters, sized and positioned exactly as in the placeholder display. The global letter was revealed by removing some of the local figure eights from the global figure eight. At the same time, each local letter was revealed by removing some of the strokes in the corresponding local figure eight. The global letter was either $\mathrm{H}$ or $\mathrm{S}$ on every trial. On each trial, all of the local letters were the same, either Es or Us, except one. In neutral response-compatibility trials, the unique letter was the other response-neutral letter ( $E$ if the nonunique local letters were Us, $U$ if the nonunique local letters were Es). In compatible trials, the unique local letter was the same as the global letter. In incompatible trials, the unique letter was the other possible global letter ( $\mathrm{S}$ if the current global letter was $\mathrm{H}$; $\mathrm{H}$ if the current global letter was S).

The position of the unique local letter was chosen so that (1) it was not in the top or bottom row of the global letter, and (2) it 
was part of both the $\mathrm{H}$ and the $\mathrm{S}$ global letters. These constraints yielded six possible locations: the four positions in the middle horizontal stroke, the position to the left and just above the horizontal stroke, and the position to the right and just below the horizontal stroke.

What has been described so far characterizes the displays in the baseline condition (Figure 4a). The onset condition differed from the baseline condition in the following ways (see Figure 4b). In the placeholder display, a local figure eight was missing in the location at which the unique letter would appear in the target display. Thus the global figure eight appeared to have a gap in it, and the onset of the unique local letter filled the gap in the target display. The restrictions on the position of the unique local letter were the same as described in the baseline condition; the position of the gap in the placeholder display was therefore uninformative about which target would appear.

The motion condition differed from the baseline condition as follows (see Figure 4c). When the target display appeared, the unique local letter began moving and continued to do so until the subject responded. If the local letter appeared in a vertical stroke of the global letter, it oscillated horizontally. The letter appeared $0.08^{\circ}$ to the left of its base position in one frame, $0.08^{\circ}$ to the right of its base position in the next frame, back in the left position in the next frame, and so on. If the local letter was in one of the two positions closest to fixation, it oscillated vertically. The letter appeared $0.06^{\circ}$ above its base position in one frame, $0.06^{\circ}$ below its base position in the next frame, in its high position in the next frame, and so on. The frame duration during motion was $100 \mathrm{msec}$.

\section{Design}

Target identity ( $\mathbf{H}$ and $\mathbf{S}$ ), response compatibility of the unique local letter (compatible, incompatible, and neutral), and temporal profile of the unique local letter (baseline, motion, and onset) were completely crossed within blocks. $H$ and $S$ appeared equally often as targets, each of the compatibility factors occurred equally often, and each of the three temporal profiles occurred equally often. The subjects completed nine blocks of 54 trials each. The order of trial presentation was randomized within each block.

\section{Procedure}

On every trial, the subject responded to the identity of the global letter. All subjects pressed the left button if the global letter was $\mathrm{H}$ and the right button if the global letter was $\mathrm{S}$.

The subjects participated for one session that lasted about $45 \mathrm{~min}$. The subjects were told to respond as quickly as possible while maintaining high accuracy. They were also told that when each trial began they should fixate on the crosshairs. The instructions were followed by a practice block of 13 trials. Each block began with four warmup trials. Each trial to which no response or an incorrect response was made was followed by a feedback tone and a single recovery trial, chosen randomly from the trials constructed for the block. Data for practice, warmup, and recovery trials were not analyzed. There was a short break after each block, lasting a minimum of $15 \mathrm{sec}$. The break after the fifth block was at least $2 \mathrm{~min}$.

When three errors were made in a block, a message was displayed which suggested that the subject should slow down to improve accuracy. The message was repeated whenever another three errors were accumulated within the same block. At the end of each block, the subjects were shown a summary of their speed and accuracy for that block.

\section{Results and Discussion}

The data were first analyzed with distance of the unique local letter from fixation included as a factor. There was no effect of distance and no interaction between distance and any other factor, so the results presented here are collapsed across distances for clarity.

Mean RTs for each temporal profile (baseline, motion, and onset) and response-compatibility condition (compatible, neutral, and incompatible) are shown in Figure 5. An ANOVA that included two factors, temporal profile and response compatibility (compatible, neutral, or incompatible), was conducted for the RTs. There were significant main effects of temporal profile and response compatibility, and there was a significant interaction between them $[F(2,30)=8.8, F(2,30)=19.3$, and $F(4,60)=$ 5.8 , respectively]. Simple effects analyses of response compatibility within each of the temporal profile conditions separately showed that response compatibility influenced RT significantly in the onset and motion conditions $[F(2,30)=14.7$ and $F(2,30)=24.8$, respectively], but not in the baseline condition $[F(2,30)=1.3]$.

Error rates are shown in Table 5. Subjects had an average error rate of $2.0 \%$. There was no evidence of a speed-accuracy tradeoff: RT and error rate were positively correlated $[r(7)=0.84, p<.05]$.

It appeared that incompatible trials produced more errors than did other trials, to a greater degree for the motion and onset conditions than for the baseline condition. An ANOVA with two factors, temporal profile and response compatibility, was conducted on the error rates. There was no significant effect of temporal profile $[F(2,15)$ $=1.4]$, but there was a significant effect of response compatibility and there was an interaction between the two factors $[F(2,30)=14.4$ and $F(4,60)=2.6$, respectively]. Overall, there was no evidence that error rates for motion trials were greater than error rates for onset and baseline trials. Simple effects analyses of response compatibility for each type of temporal profile showed that response compatibility influenced accuracy significantly

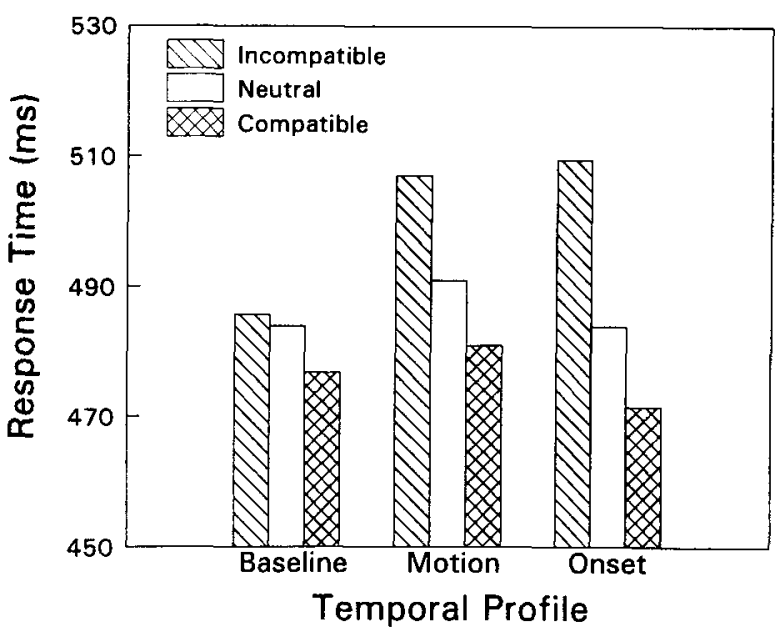

Figure 5. Results from Experiment 2. Response time is plotted as a function of response compatibility for each temporal profile of the unique local letter. 
Table 5

Error Rates (Percent) for Each Condition of Experiment 2

\begin{tabular}{lccc}
\hline & \multicolumn{3}{c}{ Response Compatibility } \\
\cline { 2 - 4 } Temporal Profile & Incompatible & Neutral & Compatible \\
\hline Baseline & 1.9 & 0.9 & 1.3 \\
Onset & 3.8 & 0.8 & 0.6 \\
Motion & 3.6 & 1.7 & 0.6 \\
\hline
\end{tabular}

in the onset and motion conditions $[F(2,30)=8.0$ and $F(2,30)=10.9$, respectively], but not in the baseline condition $[F(2,30)=1.5]$. These results for accuracy parallel the results for $\mathrm{RT}$.

In the baseline condition, no response-compatibility effects on RTs were observed. In the motion and onset conditions, the local letter's response compatibility significantly influenced responses to the global letter. This supports the hypothesis that attention is drawn to a new perceptual object in at least two ways: (1) when it appears abruptly in a previously blank location; and (2) when it segregates from its background because of relative visual motion.

It is tempting to claim that the response-compatibility effect was seen in the motion condition because motion itself captured attention. However, we reject that account, since Experiment 1 showed that motion does not capture attention. The rate of oscillation in Experiment 2 was very close to the rate used in the oscillation condition of Experiment 1 , the motion was quite salient in both experiments, and in both cases the subjects had nothing to gain by choosing to attend to the motion.

\section{EXPERIMENT 3}

Experiment 2 provided tentative support for the newobject account of attentional capture stated earlier. However, one alternative account is still viable. It is possible that the local moving element in Experiment 2 influenced responses to the global letter simply because the moving element was more salient than the stationary elements in the display (the motion stimulation sets the element apart from the remaining indistinct local letters); we will refer to this possibility as the salience hypothesis. The critical distinction between the new-object hypothesis and the salience hypothesis is whether the effects of motion on attention are transient or sustained. According to the newobject account, attention is directed to an object file when it is initially created. There is evidence that this attentional advantage dissipates within a few hundred milliseconds (Yantis \& Jones, 1991). The salience account does not make this prediction: Once the moving element becomes distinct and salient, the element should remain so throughout its motion, even if the motion itself loses its salience.

In Experiment 3, this distinction was the basis of a test between the hypotheses. The task was the same as in Experiment 2: The subjects were to identify the shape of a global letter made up of local letters. One third of the trials were baseline trials as in the baseline condition of Experiment 2 (see Figure 4a). One third included motion of a local letter beginning when the global letter appeared $1 \mathrm{sec}$ after the placeholder appeared, as in the motion condition of Experiment 2. We will call this the late-motion condition (see Figure 4c). In these trials, there is segregation of a new object while the task is being performed, so both hypotheses predict that there should be larger response-compatibility effects in the late-motion condition than in the baseline condition. The remaining trials included motion of a local letter beginning with the onset of the placeholder display. We will call this the earlymotion condition, to distinguish it from the late-motion condition (see Figure 4d). If the local letter influences performance simply because of increased salience, then the early-motion condition should yield response-compatibility effects that are as large as those in the late-motion condition. If motion captures attention only transiently, when it causes a new object to appear, then the early-motion condition should not produce response-compatibility effects that are as large as those in the late-motion condition; the attentional effect of a new object would be expected to decay by the time at which the global letter appeared.

\section{Method}

\section{Subjects}

Sixteen Johns Hopkins University students ( 8 females) participated in this experiment to fulfill research participation requirements for an introductory psychology class. No subject had participated in a related experiment before participating in this experiment.

\section{Stimuli and Design}

The stimuli in this experiment were the same as those in Experiment 2, except that the onset condition was replaced by the earlymotion condition (see Figure 4d). In these trials, motion began when the placeholder display appeared, and it continued as the letters replaced the placeholders. What was called the motion condition in Experiment 2 is called the late-motion condition in this experiment.

For all the subjects, the stimuli were displayed on a Seiko Instruments model CM1450-C color monitor controlled by a Video Seven VRAM VGA graphics card and were viewed from a distance of about $60 \mathrm{~cm}$ without the use of a chinrest.

\section{Design}

The design and procedure were identical to those of Experiment 2 , except that the early-motion condition replaced the onset condition.

\section{Results and Discussion}

Figure 6 shows mean RT as a function of temporal profile and response compatibility. An ANOVA with two factors, temporal profile (baseline, early motion, and late motion) and response compatibility (compatible, neutral, or incompatible), was conducted. There was no significant effect of temporal profile $[F(2,30)=2.2]$, but there was a significant effect of response compatibility $[F(2,30)$ $=9.9$ ]. The interaction between factors was not significant $[F(4,60)=1.8]$. Simple effects analyses of response compatibility for each temporal profile condition separately revealed a significant effect of compatibility for the late-motion condition $[F(2,30)=6.3]$, but not for the 


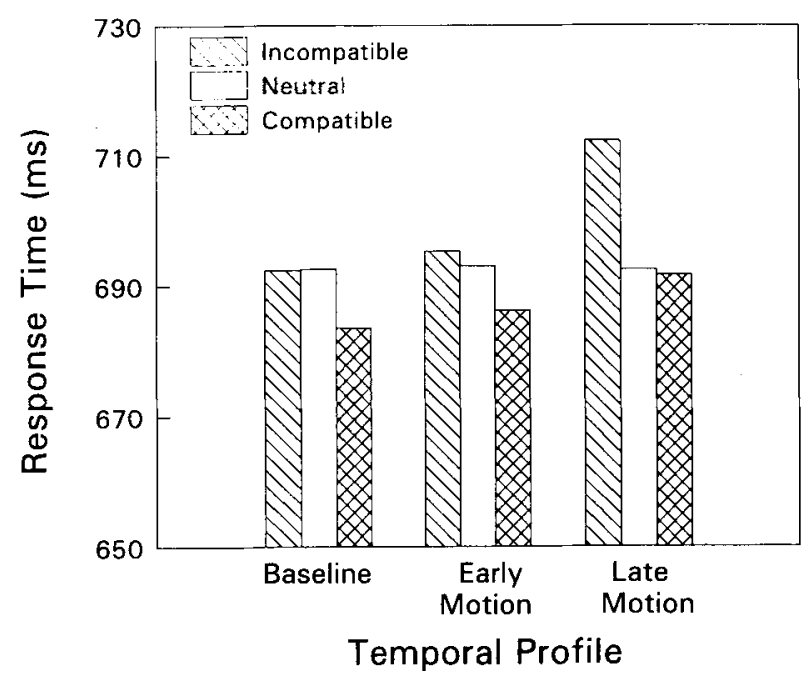

Figure 6. Results from Experiment 3. Response time is plotted as a function of response compatibility for each temporal profile of the unique local letter.

baseline condition or the early-motion condition $[F(2,30)=$ 2.3 and $F(2,30)=1.6$, respectively].

Error rates are shown in Table 6. The subjects had an average error rate of $2.6 \%$. There was no evidence of a speed-accuracy tradeoff. As responses became faster, accuracy also improved $[r(7)=0.81, p<.05]$.

The error rates were entered into an ANOVA with two factors, temporal profile and response compatibility. A significant effect of response compatibility was found $[F(2,30)=9.1]$, owing to the higher error rates for incompatible trials than for other trials. No evidence of an effect of temporal profile and no interaction between the two factors were found $[F(2,15)<1$ and $F(4,60)=2.3$, respectively]. Simple effects analyses of response compatibility for each type of temporal profile showed that response compatibility influenced accuracy significantly in the late-motion condition $[F(2,30)=13.5]$, but not significantly in the baseline and early-motion conditions $[F(2,30)=1.6$ and $F(2,30)=2.5$, respectively $]$. These results for accuracy parallel the results for RT.

In the late-motion conditions, an incompatible local element significantly slowed responses to the global letter. In the early-motion and baseline conditions, the compatibility of the local letter had no effect on responses to the global letter. This supports the hypothesis that motion draws attention to the local letter only transiently at the creation of a new object, and not because of increased salience due to motion.

Table 6

Error Rates (Percent) for Each Condition of Experiment 3

\begin{tabular}{lccc}
\hline & \multicolumn{3}{c}{ Response Compatibility } \\
\cline { 2 - 4 } Temporal Profile & Incompatible & Neutral & Compatible \\
\hline Baseline & 3.4 & 2.2 & 2.2 \\
Early motion & 2.6 & 2.9 & 1.5 \\
Late motion & 4.5 & 2.1 & 1.9 \\
\hline
\end{tabular}

The results of Experiment 2 also rule out another alternative explanation for our findings. One could argue that motion per se does capture attention, but that the visual search procedure used in Experiment 1 to test this hypothesis failed to reveal it. Contrary to this possibility, however, the late-motion condition of Experiment 3 yielded attentional capture but the early-motion condition did not. The task and the implementation of motion were the same in the two conditions; the only difference consisted in when the motion began. Only if motion captures attention transiently does the distinction between earlymotion and late-motion results make sense. But Experiment 1 is more similar in this respect to the late-motion condition of Experiment 3 than to the early-motion condition, and so the transient motion-capture explanation cannot account for all results satisfactorily. We conclude that the new-object explanation is the only account that can accommodate the findings of all three experiments.

\section{GENERAL DISCUSSION}

The experiments reported in this article seriously challenge the common belief that motion captures attention in a stimulus-driven fashion. First, we found in Experiment 1 that the same motion that can be used by an observer to guide attention efficiently when it is predictive of target location does not capture attention when it is unpredictive of target location. Experiment 2 showed that when motion segregates an element from its perceptual group, the "new object" so revealed does capture attention, and Experiment 3 showed that this occurred only transiently after the motion began, thereby ruling out the possibility that attention is captured merely by a salient figure/ground organization.

Motion joins a growing list of features that can guide attention when they constitute a target's defining attribute (e.g., Nakayama \& Silverman, 1986; Treisman \& Gelade, 1980; Treisman \& Gormican, 1988), but do not capture attention when they are irrelevant to the observer's task (see, e.g., Jonides \& Yantis, 1988; Theeuwes, 1990; Yantis, 1993a). These features are sometimes unseen when attention is focused elsewhere in the visual scene (e.g., Mack, Tang, Rock, \& Stone, 1991). Only when subjects adopt a deliberate state of attentional readiness for attributes like motion, orientation, or color singletons do such attributes guide attention (e.g., Bacon \& Egeth, in press; Folk \& Remington, in press; Folk, Remington, \& Johnston, 1992; Theeuwes, 1992; see Yantis, 1993b, and Folk, Remington, \& Johnston, 1993, for a discussion).

Together, the experiments reported here are consistent with the claim that the appearance of new perceptual objects, and little else, captures attention. According to this account, the visual system preattentively segments a visual scene (for instance, by identifying discontinuities in luminance, color, etc.) into potential objects. Usually the segmentation is unambiguous, but, as in Experiments 2 and 3 , there can be overlap in the objects when edges are ambiguous or when there are multiple levels of grouping. Object identification then proceeds in parallel, with 
attentional priority assigned to objects whose attributes most closely match those of the target. As evidence about each object's identity or attributes accumulates, priorities may be dynamically updated to reflect this partial information. When a change occurs (e.g., when something begins to move) the scene may be resegmented, and if necessary, an object file can be created for each new object (Kahneman et al., 1992). New objects are automatically assigned high attentional priority.

Two types of events have been shown to cause a new object file to be created: (1) the abrupt appearance of an object in a previously blank location (see, e.g., Yantis \& Hillstrom, 1994; Yantis \& Johnson, 1990; Yantis \& Jonides, 1984) and (2) the segregation of an object from its background when motion begins (as seen in Experiments 2 and 3). As noted by both psychologists (e.g., Kahneman \& Henik, 1981) and philosophers (e.g., Hirsch, 1982), objecthood is not disrupted (i.e., new object files are not created) by any number of reasonably continuous changes in an object's attributes (although the sorts of changes that may be considered "reasonably continuous" remain to be established).

The new-object hypothesis predicts that any feature that can cause segregation of an element from its background should also capture attention, even if that feature does not capture attention by virtue of its uniqueness alone. For example, even though irrelevant color singletons do not capture attention in standard visual search (Jonides \& Yantis, 1988), the new-object hypothesis advanced here predicts that when an element within a perceptual group changes color so that element segregates from the group as a new object, it should draw attention.

A design difference between the search experiment and the global/local experiments suggests a possible role for "event novelty" in capturing attention. In Experiment 1, there was motion on every trial; in Experiments 2 and 3 , there was motion on only one third and two thirds of the trials, respectively. It is possible to suggest that motion does capture attention in a stimulus-driven fashion, but that the regular and predictable occurrence of motion on every trial in Experiment 1 caused some sort of attentional adaptation or habituation to motion. There are at least two reasons to doubt the plausibility of this account, however. First, in Experiment 3, the unique local letter was attended in the late-motion condition but not in the early-motion condition, even though the novelty of motion was the same in both cases. Second, abrupt visual onsets are known to capture attention even when they occur on every trial. These considerations reduce the parsimony of the novelty explanation, although it is still possible that novelty does play some role in attentional capture.

Another tempting, but incomplete, explanation of our results is that motion tends to draw attention but subjects use top-down control of attention to inhibit the effect of motion. This suggestion is consistent with the general slowing of RTs in the irrelevant-motion conditions of Experiment 1 and of the motion conditions in Experiments
2 and 3. An inhibition mechanism is also consistent with the lack of capture in the early-motion condition in Experiment 3. However, the inhibition hypothesis cannot explain why motion does capture attention in the late-motion conditions in Experiments 2 and 3.

The belief that new things capture attention is as old as the belief that moving things capture attention. At the beginning of this article, we noted that William James numbered motion among the attributes that capture attention. A few pages later James (1890/1950, pp. 422-423) quoted Helmholtz (1867/1925) as follows:

The natural tendency of attention when left to itself is to wander to ever new things: and so soon as the interest of its object is over, so soon as nothing new is to be noticed there, it passes, in spite of our will, to something else.

The present experiments undermine James's observation that "moving things" capture attention involuntarily; instead, they corroborate Helmholtz's assertion that "new things" may draw attention.

\section{REFERENCES}

BACON, W. F., \& EgETH, H. E. (in press). Overriding stimulus-driven attentional capture. Perception \& Psychophysics.

Folk, C. L., \& Remington, R. W. (in press). The structure of attentional control: Contingent attentional capture by apparent motion, abrupt onset, and color. Journal of Experimental Psychology: $\mathrm{Hu}$ man Perception \& Performance.

Folk, C. L., Remington, R. W., \& Johnston, J. C. (1992). Involuntary covert orienting is contingent on attentional control settings. Journal of Experimental Psychology: Human Perception \& Performance, 18, 1030-1044.

Folk, C. L., Remington, R. W., \& Johnston, J. C. (1993). Attentional control settings: A reply to Yantis (1993[b]). Journal of Experimental Psychology: Human Perception \& Performance, 19, $682-685$

HeLmholtz, H. von (1925). Handbook of physiological optics (J. P. C. Southall, Trans.). Rochester, NY: The Optical Society of America. (Original work published 1867)

HiRsch, E. (1982). The concept of identity. New York: Oxford University Press.

JAMES, W. (1950). The principles of psychology (Vol. 1). New York: Dover. (Original work published 1890)

JoNiDEs, J. (1981). Voluntary versus automatic control over the mind's eye's movements. In L. B. Long \& A. D. Baddeley (Eds.), Attention and performance IX (pp. 187-203). Hillsdale, NJ: Erlbaum.

JoNidEs, J., \& YANTIS, S. (1988). Uniqueness of abrupt visual onset in capturing attention. Perception \& Psychophysics, 43, 346-354.

Kahneman, D., \& Henik, A. (1981). Perceptual organization and attention. In M. Kubovy \& J. R. Pomerantz (Eds.), Perceptual organization (pp. 181-211). Hillsdale, NJ: Erlbaum.

Kahneman, D., \& Treisman, A. (1984). Changing views of attention and automaticity. In R. Parasuraman \& D. R. Davies (Eds.), Varieties of attention (pp. 29-61). New York: Academic Press.

Kahneman, D., Treisman, A., \& GibBs, B. (1992). The reviewing of object files: Object-specific integration of information. Cognitive Psychology, 24, 175-219.

Kanwisher, N., \& Driver, J. (1992). Objects, attributes, and visual attention: Which, what, and where. Current Directions in Psychological Science, 1, 26-31.

Kumchi, R. (1992). Primacy of wholistic processing and global/local paradigm: A critical review. Psychological Bulletin, 112, 24-38.

Mack, A., TANG, B., Rock, I., \& Stone, W. (1991, November). Apparent motion and attention. Paper presented at the meeting of the Psychonomic Society, San Francisco. 
Mcleod, P., Driver, J., \& CrisP, J. (1988). Visual search for a conjunction of movement and form is parallel. Nature, 332, 154-155.

MilLER, J. (1981). Global precedence in attention and decision. Journal of Experimental Psychology: Human Perception \& Performance, 7, 1161-1174.

Nakayama, K. \& Silverman, G. H. (1986). Serial and parallel processing of visual feature conjunctions. Nature, 320, 264-265.

Navon, D. (1977). Forest before tress: The precedence of global features in visual perception. Cognitive Psychology, 9, 353-383.

Navon, D. (1981). The forest revisited: More on global precedence. Psychological Research, 43, 1-32.

PAQUeT, L. (1992). Global and local processing in nonattended objects: A failure to induce local processing dominance. Journal of Experimental Psychology: Human Perception \& Performance, 18, 516-529.

PAQUET, L., \& MERIKLE, P. M. (1988). Global precedence in attended and nonattended objects. Journal of Experimental Psychology: Human Perception \& Performance, 14, 89-100.

Pomerantz, J. R. (1983). Global and local precedence: Selective attention in form and motion perception. Journal of Experimental Psychology: General, 112, 516-540.

Pomerantz, J. R., \& SAGER, L. C. (1975). Asymmetric integrality with dimensions of visual pattern. Perception \& Psychophysics, 18, 460-466.

Posner, M. I., SNyder, C. R. R., \& Davidson, B. J. (1980). Attention and the detection of signals. Journal of Experimental Psychology: General, 109, 160-174.

Shulman, G. L., \& Wilson, J. (1987). Spatial frequency and selective attention to local and global information. Perception, 16, 89-101.

TheEuWEs, J. (1990). Perceptual selectivity is task dependent: Evidence from selective search. Acta Psychologica, 74, 81-99.

Theeuwes, J. (1992). Perceptual selectivity for color and form. Perception \& Psychophysics, 51, 599-606.
Treisman, A., \& Gelade, G. (1980). A feature-integration theory of attention. Cognitive Psychology, 12, 97-136.

Treisman, A., \& Gormican, S. (1988). Feature analysis in early vision: Evidence from search asymmetries. Psychological Review, 95, 15-48.

VAN DER HeJjDEN, A. H. C. (1992). Selective attention in vision. New York: Routledge.

YaNTIS, S. (1993a). Stimulus-driven attentional capture. Current Directions in Psychological Science, 2, 156-161.

YANTIS, S. (1993b). Stimulus-driven attentional capture and attentional control settings. Journal of Experimental Psychology: Human Perception \& Performance, 19, 676-681.

Yantis, S., \& Hillstrom, A. P. (1994). Stimulus-driven attentional capture: Evidence from equiluminant visual objects. Journal of Experimental Psychology: Human Perception \& Performance, 20, 1-13.

YANTIS, S., \& Johnson, D. N. (1990). Mechanisms of attentional priority. Journal of Experimental Psychology: Human Perception \& Performance, 16, 812-825.

YANTIS, S., \& JoNES, E. (1991). Mechanisms of attentional selection: Temporally modulated priority tags. Perception \& Psychophysics, 50 , 166-178.

Yantis, S., \& JoNidEs, J. (1984). Abrupt visual onsets and selective attention: Evidence from visual search. Journal of Experimental Psychology: Human Perception \& Performance, 10, 601-621.

YANTIS, S., Meyer, D. E., \& SMITH, J. E. K. (1991). Analysis of multinomial mixture distributions: New tests for stochastic models of cognition and action. Psychological Bulletin, 110, 350-374.

(Manuscript received May 11, 1993; revision accepted for publication August 30, 1993.) 